\title{
Evolution of AGN Space Densities and the Fanaroff-Riley Dichotomy
}

\author{
Melanie A. Gendre ${ }^{1}$, Jasper V. Wall ${ }^{1}$, and Philip Best ${ }^{2}$ \\ ${ }^{1}$ Department of Physics and Astronomy, University of British Columbia, 6224 Agricultural Rd, \\ Vancouver, BC, V6T 1Z1, Canada \\ Email: mgendre@phas.ubc.ca \\ ${ }^{2}$ Institute for Astronomy, Royal Observatory, Blackford Hill, Edinburgh EH9 3HJ, UK
}

\begin{abstract}
We focus on a comparison of the space densities of FR I and FR II extended radio sources at different epochs, and find that FRI and FR II sources show similar space density enhancements in various redshift ranges, possibly implying a common evolution.
\end{abstract}

Keywords. galaxies: active, galaxies: evolution, galaxies: luminosity function

Based on data compiled in the CoNFIG catalogue (Gendre \& Wall 2008; Gendre, Best \& Wall 2009), we compute the radio luminosity functions (RLF) for different redshift bins for each FR (Fanaroff \& Riley 1974) population using the $1 / V_{\max }$ technique. The FR I and FR II local $(z \leqslant 0.3)$ RLFs, in Figure 1, show apparent differences. The FR II local RLF does not show any turn-over, suggesting that there is no sharp luminosity break between FR I and FR II sources. Overall, these local RLFs indicate that locally FR I and FR II sources constitute two distinct populations. The RLF for each population in Figure 2 was then computed in different redshift bins. The overall behaviour of the enhancement with luminosity of FR I and FR II sources is very similar. With both populations showing similar enhancement history, there may be a common mechanism governing the cosmic evolution.

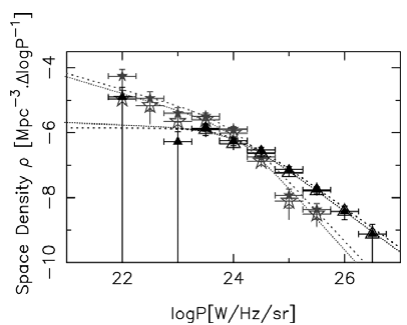

Figure 1. Local luminosity function $\rho(P)$ for FR Is and FR IIs, represented by stars and triangles respectively.
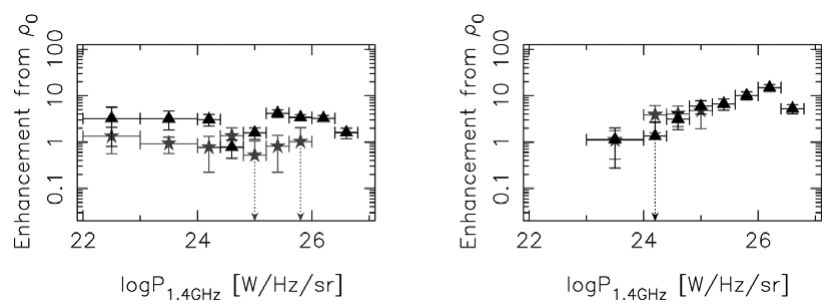

Figure 2. Comparison of the space density enhancement between FR I (stars) and FR II (triangles) sources, for different redshift bins $(0.3 \leqslant z \leqslant 0.8$ and $0.8 \leqslant z \leqslant 1.5)$.

\section{References}

Fanaroff, B. L. \& Riley, J. M. 1974, MNRAS, 167, 31P

Gendre, M. A. \& Wall, J. V. 2008, MNRAS, 390, 819

Gendre, M. A., Best, P. N., \& Wall, J. V. 2009, submitted to MNRAS 